\title{
A Stretch/Bend Method for In Situ Measurement of the Delamination Toughness of Coatings and Films Attached to Substrates
}

\section{Citation}

He, M. Y., John W. Hutchinson, and Anthony G. Evans. 2010. A stretch/bend method for In Situ measurement of the delamination toughness of coatings and films attached to substrates. Journal of Applied Mechanics 78(1): 011009.

\section{Published Version}

doi:10.1115/1.4001938

\section{Permanent link}

http://nrs.harvard.edu/urn-3:HUL.InstRepos:4895575

\section{Terms of Use}

This article was downloaded from Harvard University's DASH repository, and is made available under the terms and conditions applicable to Open Access Policy Articles, as set forth at http:// nrs.harvard.edu/urn-3:HUL.InstRepos:dash.current.terms-of-use\#OAP

\section{Share Your Story}

The Harvard community has made this article openly available.

Please share how this access benefits you. Submit a story.

\section{Accessibility}




\title{
A Stretch/Bend Method for In Situ Measurement of the Delamination Toughness of Coatings and Films Attached to Substrates
}

\author{
M. Y. He*, J.W. Hutchinson**, A. G. Evans* \\ * Materials Department, University of California, Santa Barbara, Santa Barbara, CA 93106-5050 \\ ** School of Engineering and Applied Sciences, Harvard University, Cambridge, MA 02138
}

\begin{abstract}
A stretch/bend method for the in situ measurement of the delamination toughness of coatings attached to substrates is described. A beam theory analysis is presented that illustrates the main features of the test. The analysis is general and allows for the presence of residual stress. It reveals that the test produces stable extension of delaminations, rendering it suitable for multiple measurements in a single test. It also provides scaling relations and enables estimates of the loads needed to extend delaminations. Finite element calculations reveal that the beam theory solutions are accurate for slender beams, but overestimate the energy release rate for stubbier configurations and short delaminations. The substantial influence of residual stress on the energy release rate and phase angle is highly dependent on parameters such as the thickness and modulus ratio for the two layers. Its effect must be included to obtain viable measurements of toughness. In a companion paper, the method has been applied to a columnar thermal barrier coating deposited onto a Ni-based super-alloy.
\end{abstract}

\section{Introduction}

When films and coatings are deposited onto non-planar components, methods for in situ measurement of the delamination toughness are sparse. The only viable methods have entailed indentation by cones and wedges [1,2]. But these tests are difficult to quantify. The purpose of the present article is to devise a quantitative test that has broader applicability. Its application to a thermal barrier system is described in a companion article [3]. The concept for the test is inspired by the notched four-point bend method [4, 5] (figure 1). In this test, by imposing a bending moment, a delamination extends in the coating parallel to the substrate. A steady-state region exists and the fracture toughness is ascertained from the moment that causes the delamination to extend. This test has associated mode mixity, $\psi \approx 50^{\circ}$. The implementation of this test requires a planar substrate and, often, a stiffener must be bonded to the top of the coating to assure delamination before yielding of the substrate, as depicted on figure 1 . 
The concept for the present method is depicted on figures 2 for a cylindrical substrate. However, the same concept can be applied to many different geometries, including a flat substrate (figure 3). The basic notion is that a section of the substrate be removed by electro-discharge machining (EDM), leaving an intact slender bi-layer beam comprising the substrate with attached coating. The ensuing configuration is reminiscent of the notch bend test with the distinction that the beam is supported rigidly at its ends.

For testing purposes, the specimen is placed within a tensile system with the loads applied as indicated on figure 2. By first using single-center-point loading, a throughthickness crack is introduced. Thereafter, by reverting to two-point loading, delaminations are induced that extend parallel to the substrate. The associated loads are used to provide a measure of the delamination toughness.

For general non-planar substrates a finite element method will be needed to ascertain the toughness from the loads, as illustrated in the companion article. Before embarking on such tests, guidelines for specimen design, as well as estimates of the expected loads, can be gained by invoking results generated by a beam theory analysis for a planar substrate (figure 3). Such analysis is presented in this article. Beam theory solutions are derived for the energy release rate and the compliance. The influence of residual stress is incorporated in the analysis. Thereafter, the fidelity of the results is checked using finite element analysis. Such analysis is also used to ascertain the mode mixities.

\section{Beam Theory Estimates}

\subsection{Energy Release Rates}

The clamped bi-layer beam, or wide plate, having the geometry shown in Fig. 3, comprises two homogeneous, isotropic materials. The deformations are governed by the plane strain moduli in terms of the respective Young's modulus and Poisson's ratio, $\bar{E}_{1}=E_{1} /\left(1-v_{1}^{2}\right)$ and $\bar{E}_{2}=E_{2} /\left(1-v_{2}^{2}\right)$. Prior to delamination, the bi-layer has an equilibrated residual in-plane stress $\sigma_{x x}$ in the upper layer (designated either material 1 or coating) given by

$$
\sigma_{R}(y)=\sigma_{R}^{B}\left(\frac{h_{1}+h_{2}-y}{h_{1}}\right)+\sigma_{R}^{T}\left(\frac{y-h_{2}}{h_{1}}\right), \quad h_{2} \leq y \leq h_{1}+h_{2}
$$

where $\sigma_{R}^{B}$ and $\sigma_{R}^{T}$ are stresses at the bottom and top of the upper layer, respectively. The gradient of residual stress across the upper layer is $\left(\sigma_{R}^{T}-\sigma_{R}^{B}\right) / h_{1}$. The equivalent resultant force/length and moment/length resolved about the centerline of the upper layer are:

$$
F_{R}=\frac{1}{2}\left(\sigma_{R}^{B}+\sigma_{R}^{T}\right) h_{1}, \quad M_{R}=\frac{1}{12}\left(\sigma_{R}^{T}-\sigma_{R}^{B}\right) h_{1}^{2}
$$


In addition, the clamped beam is subject to symmetrically applied transverse load/length, $P$ (Fig. 3b). The vertical displacement at the load points, $\Delta$, is measured relative to the unloaded, un-cracked beam.

The energy release rate due to $P$ and $\sigma_{R}$ can be computed based on the reduced problem in Fig. 3c characterizing changes in deformation and stress due to the delaminations. Namely, residual stress enters solely through the loads $F_{R}$ and $M_{R}$ applied to the ends of the upper layer. This linear problem will be modeled using beam (wide plate) theory under the assumption that the various members are relatively slender. The accuracy of the beam theory results will be assessed below using finite element results.

In the right half of the beam (Fig. 3c) the force/length, $F_{0}$, and moment/length, $M_{0}$, in the lower layer between the crack ends are unknown. Equilibrium requires that the moment and force in each layer are constant for $0 \leq x \leq a$. For the uncracked segment

$$
\begin{aligned}
M & =M_{0}+M^{*}, & & a \leq x \leq b \\
& =M_{0}+M^{*}+P(x-a), & & b \leq x \leq L
\end{aligned}
$$

where $M^{*}=M_{R}+F_{R} d_{1}+F_{0} d_{2}$, with $d_{1}$ and $d_{2}$ the distances of the lines of action of the forces from the neutral bending axis of the bonded bi-layer. For $x>a$, the horizontal force/length is constant: $F=F_{0}-F_{R}$.

The strain energy, $S E$, in the half-section to the right of $x=0$ in the reduced problem in Fig. $3 \mathrm{c}$ constitutes the sum of the bending and stretching energies in the two beams to the left at $x=a$ and in the bonded bi-layer to the right of the crack tip. The result is readily determined as

$$
\begin{aligned}
S E & =\frac{M_{0}^{2} a}{2 B_{2}}+\frac{M_{R}^{2} a}{2 B_{1}}+\frac{F_{0}^{2} a}{2 \bar{E}_{2} h_{2}}+\frac{F_{R}^{2} a}{2 \bar{E}_{1} h_{2}}+\frac{\left(M_{0}+M^{*}\right)^{2}(L-a)}{2 B} \\
+ & \frac{\left(M_{0}+M^{*}\right) P(L-b)^{2}}{2 B}+\frac{\left(F_{0}-F_{R}\right)^{2}(L-a)}{2\left(\bar{E}_{1} h_{1}+\bar{E}_{2} h_{2}\right)}+\frac{P^{2}(L-b)^{3}}{6 B}
\end{aligned}
$$

Here, $B_{1}=\bar{E}_{1} h_{1}^{3} / 12, B_{2}=\bar{E}_{2} h_{2}{ }^{3} / 12$ and $B$ is the bending stiffness of the bonded bilayer given by

$$
B=B_{1}+B_{2}+\bar{E}_{1} h_{1} d_{1}^{2}+\bar{E}_{2} h_{2} d_{2}^{2}
$$

with $d_{1}=h_{2}+h_{1} / 2-c, d_{2}=c-h_{2} / 2$ and

$$
c=\frac{\left(\bar{E}_{2} h_{2}^{2}+\bar{E}_{1}\left(h_{1}^{2}+h_{1} h_{2}\right)\right)}{2\left(\bar{E}_{1} h_{1}+\bar{E}_{2} h_{2}\right)} .
$$

With $P, F_{R}$ and $M_{R}$ prescribed, $S E$ in (4) is the complementary potential energy of the clamped half-beam with equilibrium being satisfied for all combinations of $F_{0}$ and $M_{0}$. Invoking the principle of minimum complementary potential energy, $F_{0}$ and $M_{0}$ can be obtained by minimizing (5) with respect to these two unknowns with the result 


$$
\begin{gathered}
M_{0}\left(\frac{a}{B_{2}}+\frac{L-a}{B}\right)+F_{0}\left(\frac{(L-a) d_{2}}{B}\right)=-\frac{P(L-b)^{2}}{2 B}-\frac{\left(M_{R}+F_{R} d_{1}\right)(L-a)}{B} \\
M_{0}\left(\frac{(L-a) d_{2}}{B}\right)+F_{0}\left(\frac{a}{\bar{E}_{2} h_{2}}+\frac{(L-a) d_{2}^{2}}{B}+\frac{L-a}{\bar{E}_{1} h_{1}+\bar{E}_{2} h_{2}}\right)=-\frac{P d_{2}(L-b)^{2}}{2 B} \\
-\frac{\left(M_{R}+F_{R} d_{1}\right) d_{2}(L-a)}{B}+\frac{F_{R}(L-a)}{\bar{E}_{1} h_{1}+\bar{E}_{2} h_{2}}
\end{gathered}
$$

Since $F_{0}$ and $M_{0}$ are linear (homogeneous of degree one) in $P, F_{R}$ and $M_{R}$, the $S E$ is a quadratic function (homogeneous of degree two) of $P, F_{R}$ and $M_{R}$, as well as being a function of the delamination crack length, $a$ :

$$
\operatorname{SE}\left(P, F_{R}, M_{R}, a\right)
$$

For prescribed $P, F_{R}$ and $M_{R}$, the energy release rate is

$$
G=\frac{\partial S E}{\partial a}
$$

The formula for $G$ is too lengthy to provide analytical insights, but it is straightforward to use numerical differentiation of (8) to evaluate $G$ by using (4) in conjunction with (6). Inspection of the formulae reveals that, in the absence of residual stress, the energy release rate can be expressed in the normalized form

$$
G \bar{E}_{2} h_{2}{ }^{3} /(P L)^{2} \equiv \Pi\left(a / L, h_{1} / h_{2}, E_{1} / E_{2}, b / L\right)
$$

In the presence of residual stress another non-dimensional group is required, given by:

$$
\mathfrak{R} \equiv \sigma_{R} h_{1}^{2} / P L
$$

Basic results are illustrated on figure 4, absent residual stress, for three different ratios of elastic properties, $E_{2} / E_{1}$. All plots are for equivalent thickness coating and substrate, as well as for loads applied at $b / L=0.5$. The two principal features are as follows.

(i) The energy release rate diminishes as the delamination extends (at fixed load). Consequently, upon equating the energy release rate to the toughness, the delamination progresses only upon increasing the load. Namely, crack extension is stable.

(ii) The energy release rate decreases as the coating becomes more compliant.

\subsection{Compliance}

The dependence of the load point deflection, $\Delta$, on $P, F_{R}$ and $M_{R}$ is obtained by noting that $S E$ be equal to the work done by the three loads for the reduced problem in Fig. 4c. To this end, let $\mathbf{F}=\left(P, F_{R}, M_{R}\right)$ and $\mathbf{U}=(\Delta, u, \theta)$, where $u$ is the displacement 
through which $F_{R}$ works and $\theta$ is the rotation through which $M_{R}$ works. Introduce the $3 \times 3$ symmetric compliance matrix, $\mathbf{C}$, such that $\mathbf{U}=\mathbf{C F}$. For any loading sequence with $a$ fixed,

$$
\frac{1}{2} \mathbf{F} \cdot \mathbf{U}=\frac{1}{2} \mathbf{F}^{T} \mathbf{C F}=S E
$$

Due to the quadratic dependence of $S E$ on $\mathbf{F}$ it follows that $C_{i j}=\partial^{2} S E / \partial F_{i} \partial F_{j}$. In particular, with

$$
\Delta=C_{11} P+C_{12} F_{R}+C_{13} M_{R},
$$

the coefficients are

$$
C_{11}=\frac{\partial^{2} S E}{\partial^{2} P}, C_{12}=\frac{\partial^{2} S E}{\partial P \partial F_{R}}, C_{13}=\frac{\partial^{2} S E}{\partial P \partial M_{R}}
$$

These compliances, which depend on $a$ but not on $\mathbf{F}$, are also readily computed using numerical differentiation with (4) and (6). Standard formulas for second order partial derivatives provide exact results due to the quadratic dependence of $S E$ on $\mathbf{F}$. The residual stresses contribute to the load-point displacement, $\Delta$, due to coupling between the transverse loads and the residual stresses in the cracked beam.

These compliances can be used to ascertain the delamination length by periodic, partial unloading at various stages during crack extension.

\subsection{Influence of Residual Stress}

The residual stress influences the energy release rate in a complicated manner because of its non-linear interaction with other parameters in the problem. Consequently, for this article, they are explored within a limited parameter set. Namely, results are presented for a system having the characteristics described in the companion article: modulus ratio, $E_{2} / E_{1} \approx 4$ and thickness ratio in the range, $1 \leq h_{1} / h_{2} \leq 3$. To clarify some of the features, the results are presented using two schemes: $\Pi(1 / \mathfrak{R})$ and $\Pi(\mathfrak{R})$.

Results for $\Pi(\mathfrak{R})$ at two different thickness, $h_{1} / h_{2}=1,3$ are plotted on figure 5a, covering a wide range of residual stress from tensile (positive) to compressive (negative). The trends for the equi-thickness beams are rationalized in a straightforward manner. Namely, because the applied load places layer \#1 into tension and layer \#2 into compression, a tensile residual stress in layer \#1 would be expected to elevate the stress and thus, the energy release rate, and vice versa, as evident in the plots.

The results for $h_{1} / h_{2}=3$ are more surprising (figure $5 \mathrm{~b}$ ). They are also the more relevant to the interpretation of the tests in the companion article. For the longer delaminations, $\mathrm{a} / \mathrm{L}>0.3$, the energy release increases regardless of the sign of the residual stress. The characteristics become more apparent in the $\Pi(1 / \mathfrak{R})$ plot (figure $6 \mathrm{a})$. This reveals that, at $1 / \mathfrak{R} \geq 20$, the energy release rate asymptotes to the solution absent residual stress. Moreover, at the higher residual stress, $\Pi$ can be fit by the parabolic formula: 


$$
\begin{aligned}
& \Pi \approx \Pi_{0}+\Delta \Pi \\
& \Delta \Pi=\left(\mathfrak{R} / \mathfrak{R}_{0}\right)^{2}
\end{aligned}
$$

where (for the parameter set chosen and $\mathrm{a} / \mathrm{L}=0.4$ ) $\Pi_{0} \approx 4.1 \times 10^{-4}$ and $\Re_{0}=7.1$. Converting (11a) back into dimensional terms, the energy release rate becomes,

$$
G \approx \frac{\Pi_{0}(P L)^{2}}{E_{2} h_{2}^{3}}+\frac{1}{\mathfrak{R}_{0}^{2}}\left(\frac{h_{1}}{h_{2}}\right)^{3} \frac{\sigma_{R}^{2} h_{1}}{E_{2}}
$$

Namely, $G$ is essentially the simple addition of the contributions from the load (first term) and the residual stress (second term). This is surprising since, not only is it not normally possible to add energy release rates but also the elevation in $G$ regardless of sign is unexpected. We are not able to provide a compelling argument for this behavior except to invoke the rapid change in phase angle that occurs as the residual stress increases, as elaborated below.

Equating $G$ in (11a) to the delamination toughness, $\Gamma$, the load required for crack extension becomes:

$$
P_{c}=\frac{1}{\Pi_{0}}\left(\frac{h_{2}}{L}\right) \sqrt{\Gamma E_{2} h_{2}}\left[1-\left(\frac{\sigma_{R}^{2}}{\Gamma E_{2}}\right) \frac{h_{1}^{4}}{h_{2}^{3} \mathfrak{R}_{0}^{2}}\right]
$$

Evidently, as the residual compression increases, the delamination extends at lower load, regardless of the sign.

The behavior for shorter delaminations is more nuanced (Figures $5 \mathrm{a}$ and $6 \mathrm{~b}$ ), especially in the range wherein $\Pi$ decreases as $1 / \Re$ decreases. In this range, the rate of change of $\Pi$ with $\mathfrak{R}$ dictates that the delamination load, $P_{c}$, increases as the residual compression increases analogous to the behavior in equi-thickness systems (figure 5a). A detailed interpretation has not been pursued.

\section{Finite Element Comparison}

A finite element analysis has been conducted for the configurations that duplicate the results presented on figures 4 and 5 . The analysis is conducted using the finite element code ABAQUS. The energy release rates are calculated as well as the mode mixity using standard procedures [6]. When the results are superposed on figure 4, it becomes apparent that the beam theory solution is accurate (to within $10 \%$ ) provided that the delamination length is sufficiently large, $a / L \geq 0.2$ and that the beams are slender, $L / h>10$. Calculations conducted in the presence of residual compressive stress are superposed onto figure 6 . The implications are essentially the same. Namely, the beam theory results are accurate except for stubby beams with short delaminations. Often, practical limitations require that tests be performed with dimensions and properties 
outside this range. When such circumstances apply, toughness levels must be ascertained from the measurements by means of numerical results. An illustration is presented in the companion article.

The mode mixities, $\Psi$, corresponding to these energy release rates are illustrated for long delaminations on figure 7 . For this configuration, there is a rapid change in $\Psi$ with residual stress that is almost symmetric about zero. Namely, for large residual compression, the delamination is almost mode II $\left(\Psi \approx 80^{\circ}\right)$, this angle systematically diminishes as the stress deceases and is about mode $\mathrm{I}\left(\Psi \approx 0^{0}\right)$ when the stress is zero. Then, when the stress becomes tensile the angle increases again, but now with opposite sign, and approaches mode II again at high stress. It should be emphasized that this symmetry is specific to the parameters used in the analysis. Beams with different choices of the thickness and modulus ratios and delamination lengths would not behave in the same manner.

The following interpretation is suggested by the basic mechanics of the relative contributions to the mode I and II stress intensities $\left(K_{I}, K_{I I}\right)$ from the axial force/width, $p$, and bending moment/width, $m$, associated with the stress state in layer \#1. In the simplest manifestation (neglecting the contributions from the substrate) [7]:

$$
\begin{aligned}
& K_{I}=0.44 p h_{i}^{-1 / 2}+2.7 m h_{1}^{-3 / 2} \\
& K_{I I}=0.56 p h_{i}^{-1 / 2}-2.1 m h_{1}^{-3 / 2}
\end{aligned}
$$

For thin substrates, the neutral axis due to the applied load resides in layer \#1, causing the contributions to $K_{I}$ and $K_{I I}$ from $p$ and $m$ to be comparable. Consequently, in the absence of residual stress, for the parameters applicable to figure $7, K_{I I} \approx 0$ is negative and $K_{I}$ is positive. The residual stress, being spatially uniform, does not generate a moment. It contributes only through $\mathrm{p}$ (positive for tension and negative for compression). Accordingly, $K_{I I}$ becomes non-zero and increases as the residual stress increases, with different sign for tension and compression. This feature is apparent in figure 7. That the energy release rate created by the applied load is essentially mode I while that from the residual stresses is largely mode II is believed to be the basis for the additive nature of the energy release rates implicit in (11).

\section{Concluding Remarks}

A test has been designed that can be used to measure in situ the delamination toughness of coatings or films attached to components. This is achieved by removing a section of the substrate by EDM, leaving a slender coating/substrate bi-layer attached to the remainder of the component. When loaded, the bi-layer behaves as a beam rigidly supported at its ends. Analysis of the test is presented for a planar system, whereupon beam theory can be used to estimate the energy release rates and compliances. Such results, derived with and without residual stress, facilitate specimen design and provide estimates of the loads needed to extend delaminations. For actual configurations, which 
may be non-planar, finite element analysis would be needed to ascertain the toughness from the loads, as exemplified in a companion paper.

The beam theory results reveal that the test produces stable extension, rendering it suitable for multiple measurements on a single test. Namely, the load needed to continue extension of the delamination, $P_{c}$, increases as it lengthens. Finite element calculations affirm that these solutions are accurate for slender beams and long delaminations, but overestimate the energy release rate for stubbier configurations and short delaminations. The change in the energy release rate due to residual compression in the coating has been explicitly addressed for long delaminations. The results reveal that $P_{c}$ always decreases as the residual stress increases, despite the requirement that, for the crack to extend, the tensile strain due to the bending must first overcome the residual compression.

\section{References}

1. M. R. Begley, D. R. Mumm, A. G. Evans, and J. W. Hutchinson, Analysis of a Wedge Impression Test for Measuring the Interface Toughness Between Films/Coatings and Ductile Substrates, Acta mater., 48, 3211-3220 (2000).

2. M. J. Stiger, R. Handoko, J. L. Beuth, F. S. Pettit and G. H. Meier, Accelerated durability testing of gas turbine Coatings Emphasizing Oxide-metal interfaces, Long Term Durability of Structural Materials, (2001) 207-219.

3. C. Eberl, D. S. Gianola, K. Hemker, M. Y. He, A. G. Evans, Manuscript in preparation.

4. P. G. Charalambides, J. Lund, A. G. Evans and R. M. McMeeking, A Test Specimen for Determining the Fracture Resistance of Bimaterial Interfaces, P. G. Charalambides, J. Lund, A. G. Evans and R. M. McMeeking, J. Appl. Mech. 111 March, 77-82 (1989)

5. P. Charalambides, H.C. Cao, J. Lund and A.G. Evans, Development of a Test Method for Measuring the Mixed Mode Fracture Resistance of Bimaterial Interfaces, P. Charalambides, H.C. Cao, J. Lund and A.G. Evans. Mech. of Materials [8] 269-283 (1990)

6. ABAQUS Version 6.7, Dassault Systemes, 2007

7. J. W. Hutchinson, Z. Suo, Mixed Mode Cracking in Layered Materials, Hutchinson, J.W., Suo, Z., Adv. Appl. Mech., 29 (1992) 63-191.

\section{Figures}

Figure 1. A schematic of the notched four point bend test for determining delamination toughness, indicating the location of bonded stiffeners often need to assure delamination before substrate yielding.

Figure 2. (a) A schematic of the specimen design and test procedure for the example of a coating on the periphery of a circular substrate. The pre-cracking method is illustrated on 
the top right and the subsequent two-point loading for ascertaining the delamination toughness is on the top left. (b) An optical image of the actual configuration used in the companion paper comprising a columnar thermal barrier coating deposited onto a Nibased alloy.

Figure 3. Diagrams illustrating the mechanics methods used to obtain the energy release rate on a planar system with residual stress.

Figure 4. Beam theory predictions of the energy release rate as a function of delamination length for cases with zero residual stress. Also shown are selected finite element results for various levels of the relative span, $h / L$. All of the results are for equi-thickness layers, $h_{2} / h_{1}=1$ and for a loading span, $b / L=0.5$ but differing modulus ratios, as indicated on the figures.

Figure 5. Beam theory predictions of the energy release rate as a function of the level of residual stress in the coating for two different thickness ratios. (a) $h_{1} / h_{2}=1$, (b) $h_{1} / h_{2}=3$. All of the results are for $E_{1} / E_{2}=4$ and a loading span, $b / L=0.5$.

Figure 6. Beam theory predictions of the energy release rate as a function of the inverse residual compression in the coating for two different delamination lengths: (a) $a / L=0.4$ and (b) $\mathrm{a} / \mathrm{L}=0.1$. Also shown are selected finite element results for various levels of the relative span, $h / L$. All of the results are for $h_{1} / h_{2}=3, E_{1} / E_{2}=4$ and for a loading span, $b / L=0.5$.

Figure 7. The mode mixity (phase angle) as a function of the residual stress. The results are for $\mathrm{a} / \mathrm{L}=0.4, h_{1} / h_{2}=3, E_{1} / E_{2}=4$ and for a loading span, $b / L=0.5$. 


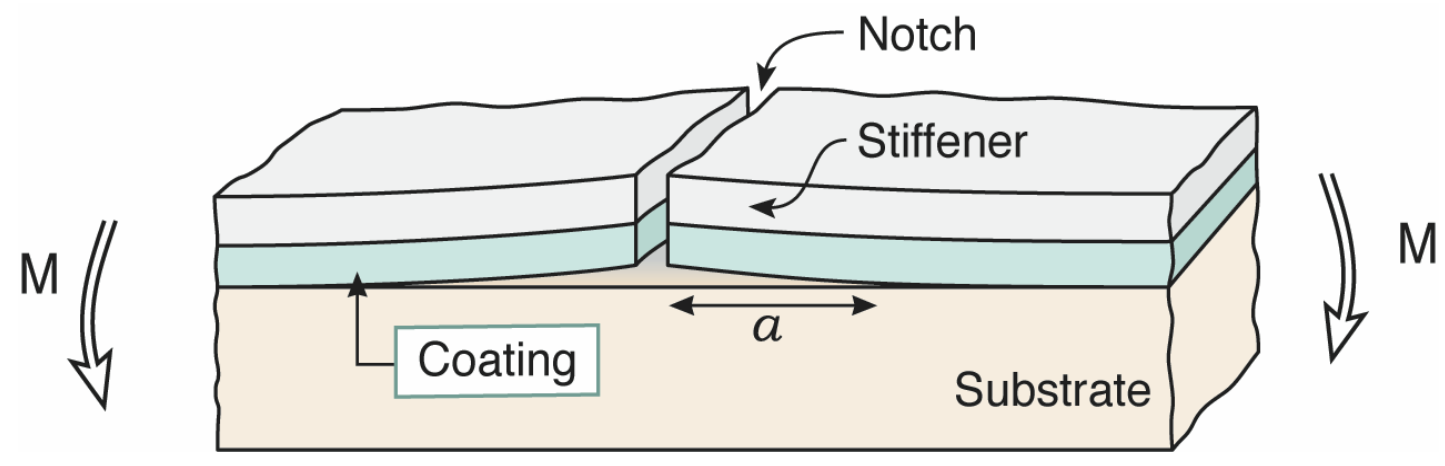

Figure 1. A schematic of the notched four point bend test for determining delamination toughness, indicating the location of bonded stiffeners often need to assure delamination before substrate yielding. 


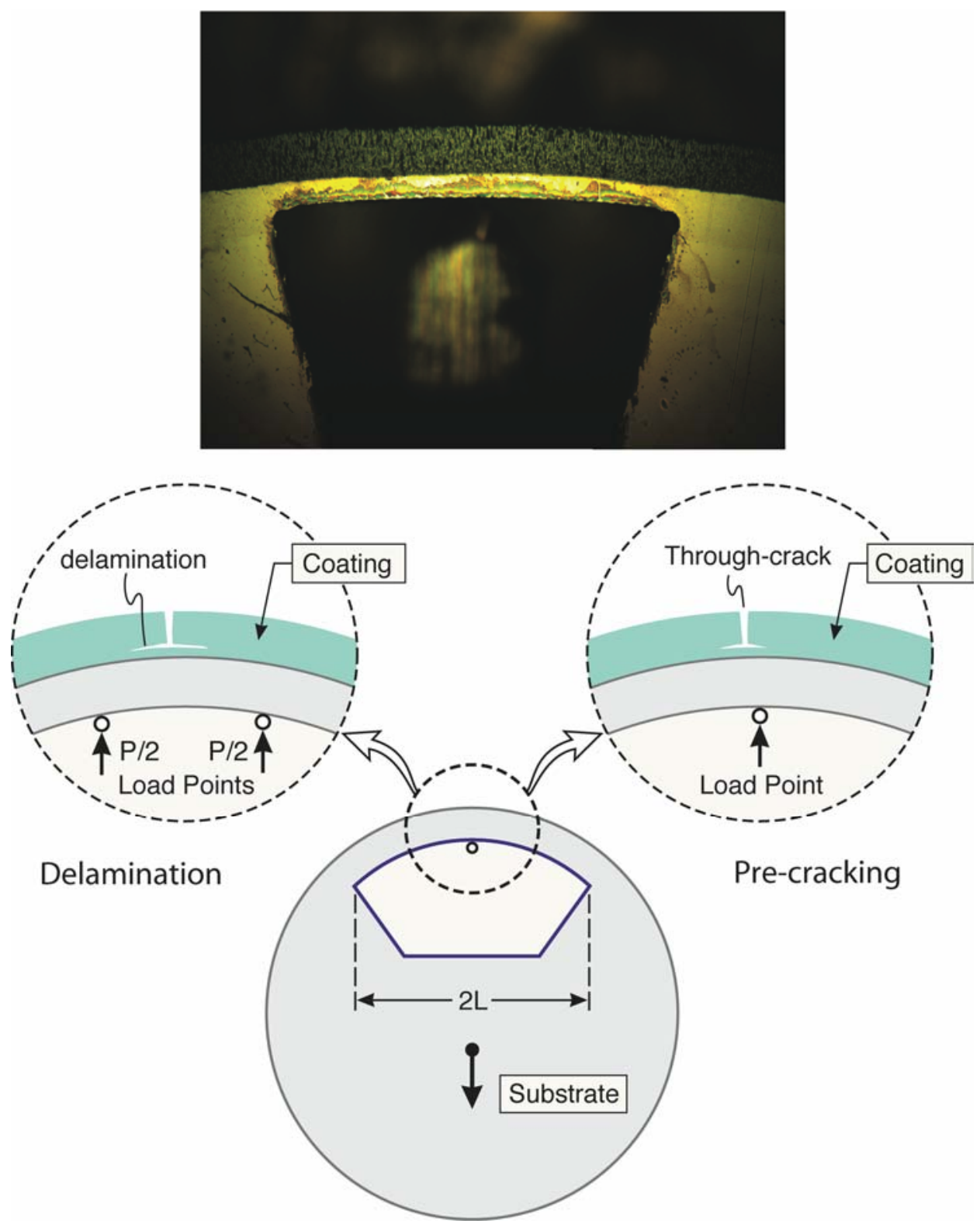

Figure 2. (a) A schematic of the specimen design and test procedure for the example of a coating on the periphery of a circular substrate. The pre-cracking method is illustrated on the top right and the subsequent two-point loading for ascertaining the delamination toughness is on the top left. (b) An optical image of the actual configuration used in the companion paper comprising a columnar thermal barrier coating deposited onto a $\mathrm{Ni}$ based alloy. 
a

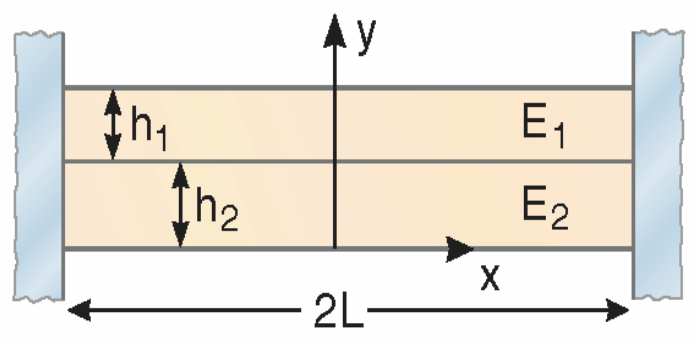

b

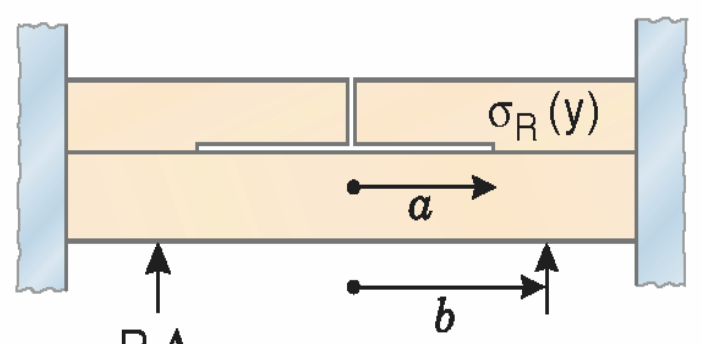

$\mathrm{P}, \Delta$

C

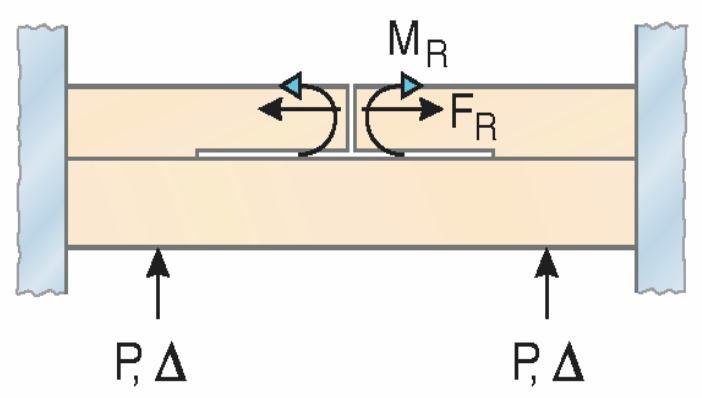

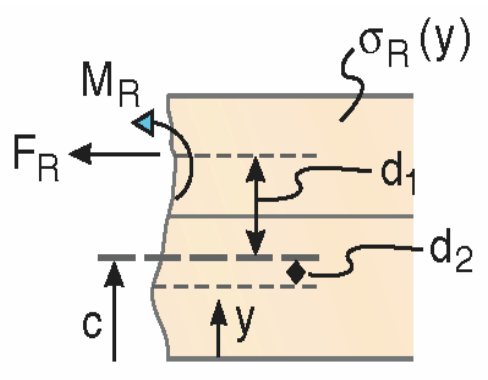

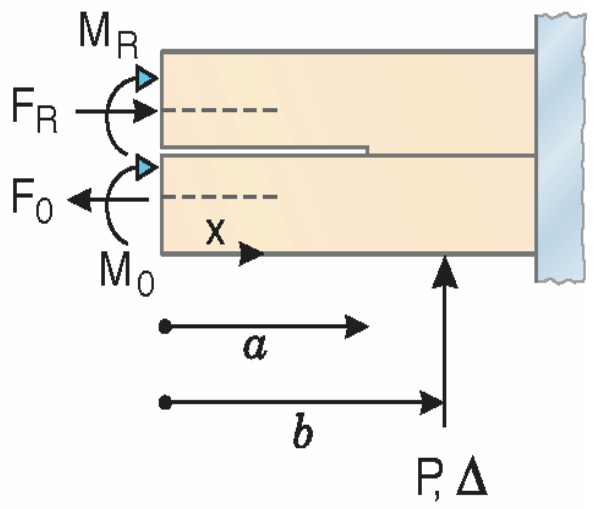

Figure 3. Diagrams illustrating the mechanics methods used to obtain the energy release rate on a planar system with residual stress 


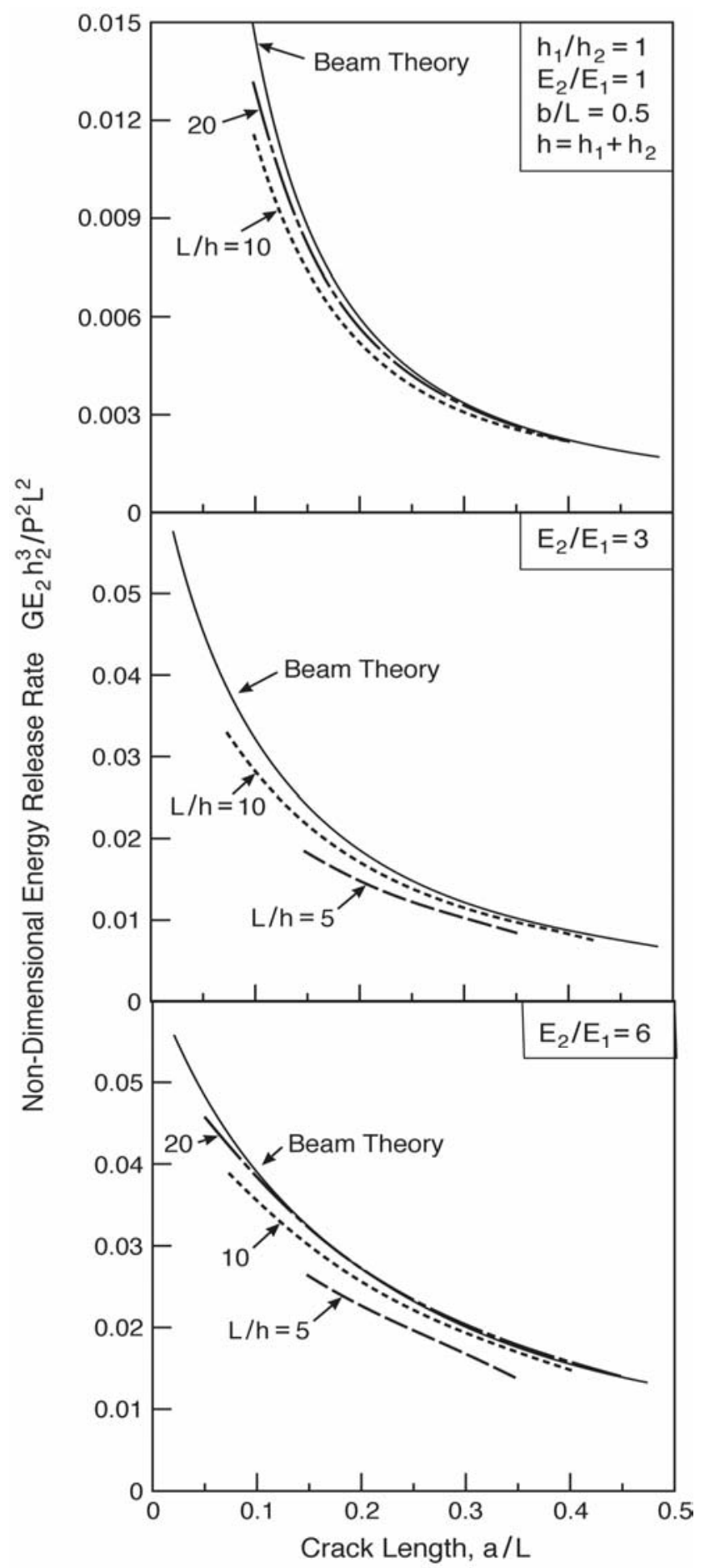

Figure 4. Beam theory predictions of the energy release rate as a function of delamination length for cases with zero residual stress. Also shown are selected finite element results for various levels of the relative span, $h / L$. All of the results are for equi-thickness layers, $h_{2} / h_{1}=1$ and for a loading span, $b / L=0.5$ but differing modulus ratios, as indicated on the figures. 

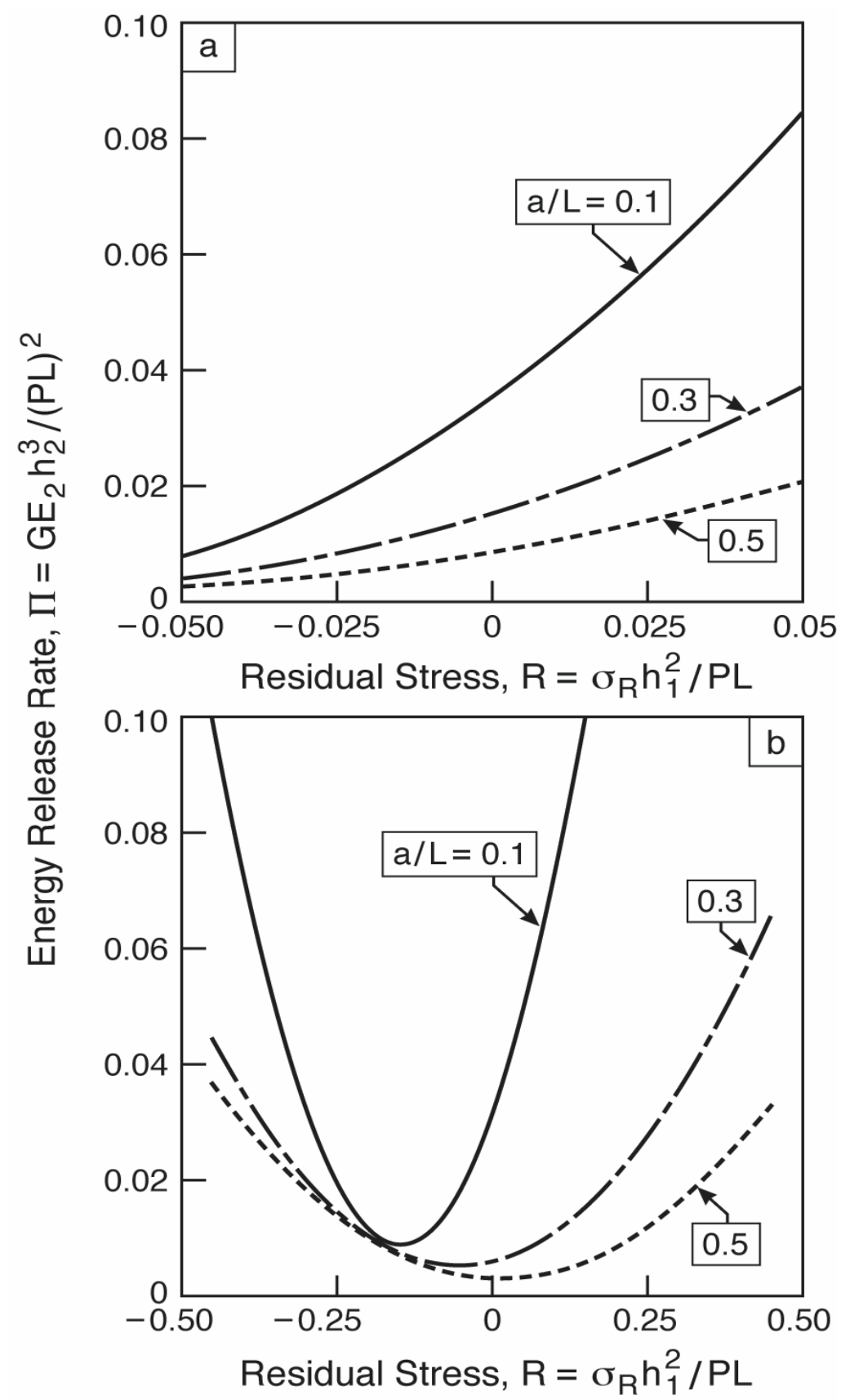

Figure 5. Beam theory predictions of the energy release rate as a function of the level of residual stress in the coating for two different thickness ratios. (a) $h_{1} / h_{2}=1$, (b) $h_{1} / h_{2}=3$. All of the results are for $E_{1} / E_{2}=4$ and a loading span, $b / L=0.5$. 


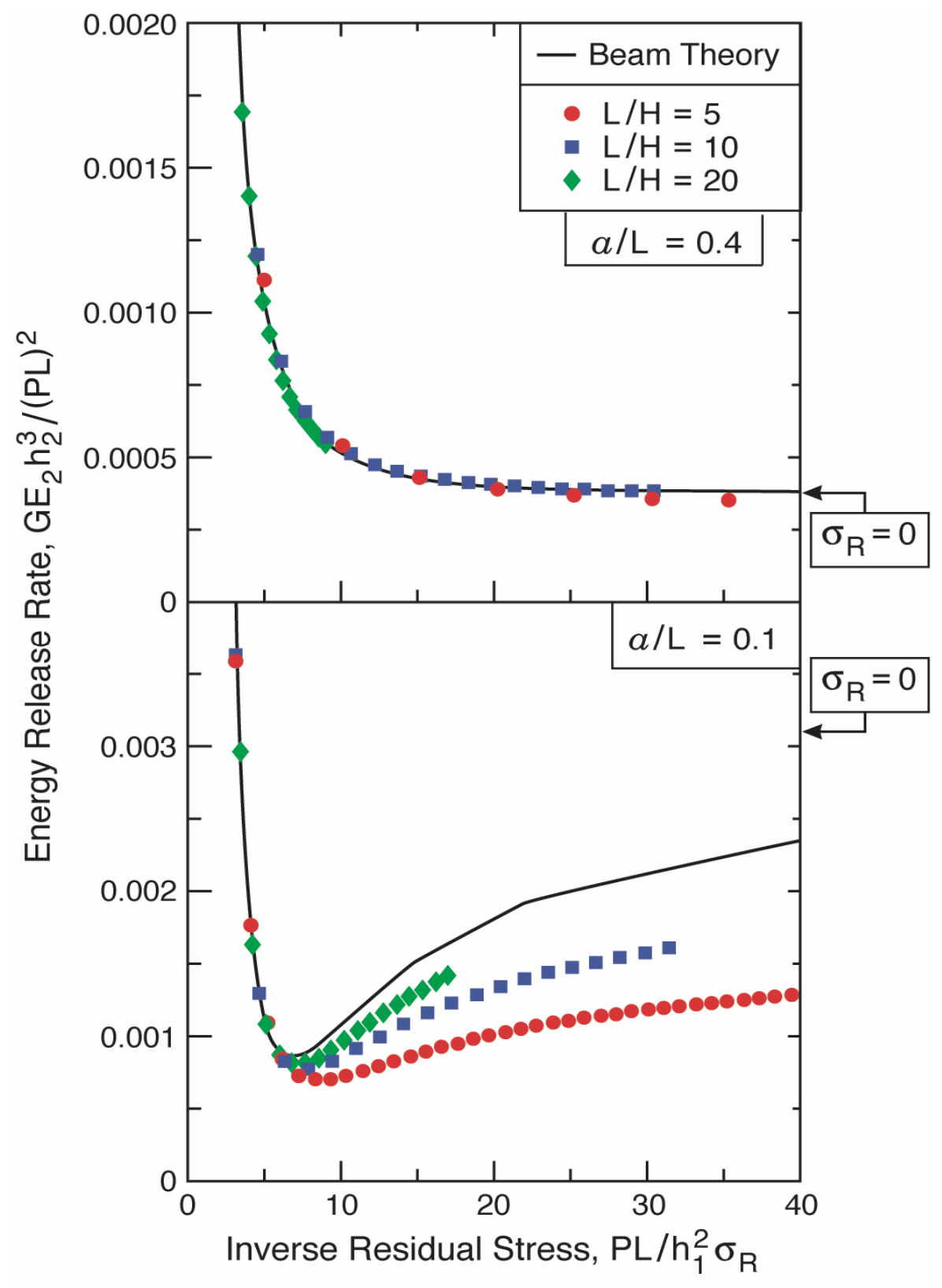

Figure 6. Beam theory predictions of the energy release rate as a function of the inverse residual compression in the coating for two different delamination lengths: (a) a/ $\mathrm{L}=0.4$ and (b) $\mathrm{a} / \mathrm{L}=0.1$. Also shown are selected finite element results for various levels of the relative span, $h / L$. All of the results are for $h_{1} / h_{2}=3, E_{1} / E_{2}=4$ and for a loading span, $b / L=0.5$. 


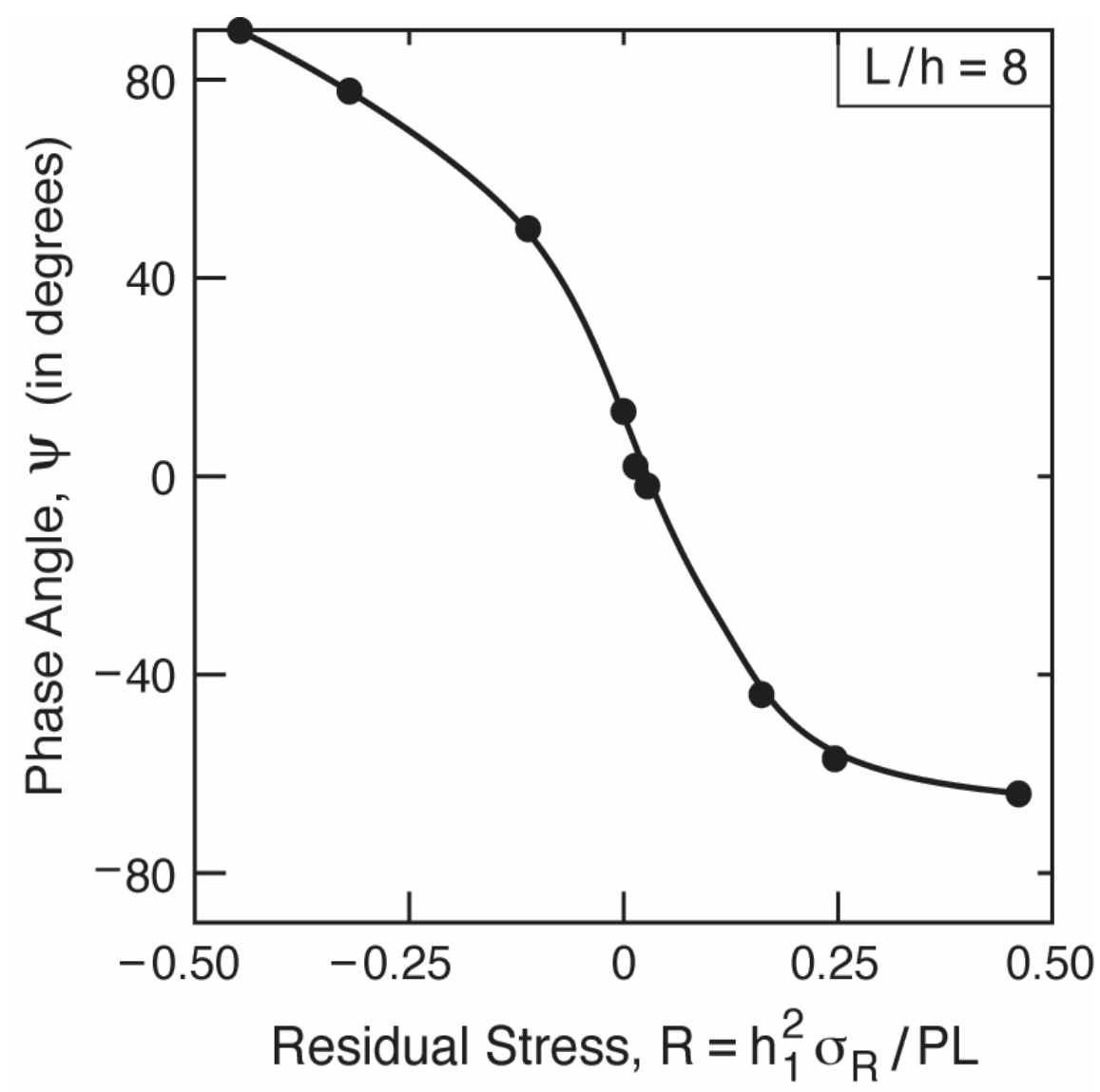

Figure 7. The mode mixity (phase angle) as a function of the residual stress. The results are for $\mathrm{a} / \mathrm{L}=0.4, h_{1} / h_{2}=3, E_{1} / E_{2}=4$ and for a loading span, $b / L=0.5$. 